\title{
Controlling and tuning strong optical field gradients at a local probe microscope tip apex
}

\author{
Olivier J. F. Martin ${ }^{\mathrm{a})}$ \\ Laboratory for Field Theory and Microwave Electronics, Swiss Federal Institute of Technology, \\ ETH-Zentrum, 8092 Zurich, Switzerland \\ Christian Girard \\ Laboratoire de Physique Moléculaire URA CNRS 772, Université de Franche Comté, 25030 Besançon, \\ France
}

(Received 7 May 1996; accepted for publication 4 December 1996)

\begin{abstract}
We show that strong optical field gradients can be created at the tip apex of a local probe microscope illuminated by an external light source. We demonstrate that these confined fields can be easily, precisely and continuously tuned by changing the polarization and the incidence of the external field. We also investigate the topology of the field intensity in the tip-surface junction. (C) 1997 American Institute of Physics. [S0003-6951(97)03206-3]
\end{abstract}

Recent developments of local probe microscopes [scanning tunneling microscope, (STM), atomic force microscope (AFM), and scanning near-field optical microscope (SNOM)] have generated considerable interest in the understanding of the interaction of a local probe microscope tip with an external illuminating field. In fact, this interaction does not only play a central role in apertureless SNOM, ${ }^{1-7}$ but has also been implemented in local probe assisted nanoengineering, as for example in STM assisted nano-lithography. ${ }^{8}$

A typical geometry for this problem is depicted in Fig. 1. A local probe tip, placed at a very small distance from a surface is illuminated by an external field $\mathbf{E}^{0}$. The generation of strong field gradients in such a tip-surface junction has been studied by Denk and Pohl. ${ }^{9}$ Using a quasistatic model, these authors have investigated in detail the influence of the material parameters on the field in the junction. In particular, they have demonstrated that extremely strong field gradients can be produced at the tip apex, when the excitation wavelength is such that plasmon resonances can be excited.

In this letter, we will show that it is also possible to create strong optical field gradients in such a junction without having recourse to plasmon resonances. We will also investigate the influence of two important experimental parameters on the field in the junction; namely the polarization and the incidence of the external excitation.

The geometry used for our calculations is depicted in Fig. 1. We consider a tetragonal tungsten tip placed above a perfectly flat silica surface. The interface silica-air is located at $z=0$ and the tip apex at $(x, y, z)=(0,0,5)[\mathrm{nm}]$.

This tip-surface junction is illuminated by an external plane wave propagating along the $\mathbf{k}$ direction, so that it impinges on the tip with an angle $\varphi$. Throughout this letter, we consider an incident field impinging on the edge of the tetragonal tip $\left(\varphi=45^{\circ}\right)$ and allow for incident angles $\theta$ between $40^{\circ}$ and $90^{\circ}$.

The incident field $\mathbf{E}^{0}$ is linearly polarized and its intensity equal to unity. We consider a wavelength of $633[\mathrm{~nm}]$. At this wavelength the relative dielectric constant of tungsten and silica are, respectively, $\varepsilon=(4.8 ; 21.2)$ (Ref. 10) and

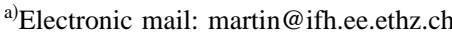

$\varepsilon=(2.4 ; 0.0) .{ }^{11}$ Therefore, no plasmon resonance can occur in that system.

For a given propagation direction $\mathbf{k}$, the polarization angle $\psi$ of the incident field $\mathbf{E}^{0}$ can be varied between $0^{\circ}$ and $90^{\circ}$ (Fig. 1). A polarization angle $\psi=0^{\circ}$ corresponds to $\mathrm{p}$ polarization $\left(\mathbf{E}^{0} \| \mathbf{u}_{\mathrm{p}}\right)$, whereas $\psi=90^{\circ}$ corresponds to s polarization $\left(\mathbf{E}^{0} \| \mathbf{u}_{\mathrm{s}}\right)$. Let us emphasize that the polarization angle $\psi$ can be continuously and precisely varied experimentally between $0^{\circ}$ and $90^{\circ}$.

To compute the field in the junction, we use the generalized field propagator technique. ${ }^{12}$ This three-dimensional vectorial formalism provides a self-consistent solution for the field in complex geometries. Furthermore, it enables one to obtain in a single calculation the responses of the system to many different incident fields. Thus different illumination parameters such as polarization and angle of incidence may be readily investigated. The tip used for the calculation has a volume of $1.7 \cdot 10^{6}\left[\mathrm{~nm}^{3}\right]$ and is discretized with a 2593 mesh adaptive grid (with higher refinement at the apex). The radius of curvature of the very tip is $0.5[\mathrm{~nm}]$.

In Fig. 2 we study the influence of the incident polarization on the total field intensity $I$ in the tip-surface junction. For p polarization $\left(\psi=0^{\circ}\right)$, we observe extremely strong field gradients just below the tip apex, where $I$ reaches 183

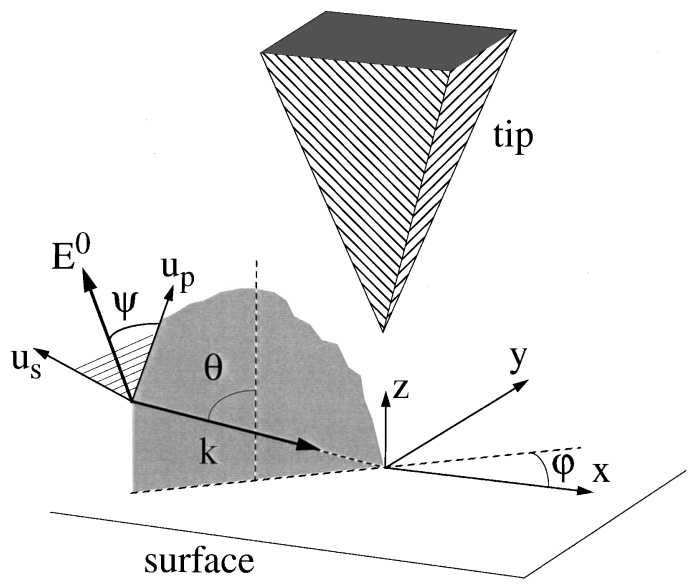

FIG. 1. Geometry used for our calculations. The tip-surface junction is illuminated by an external plane wave $\mathbf{E}^{0}$ propagating along $\mathbf{k}$-direction and polarized with an angle $\psi$. 

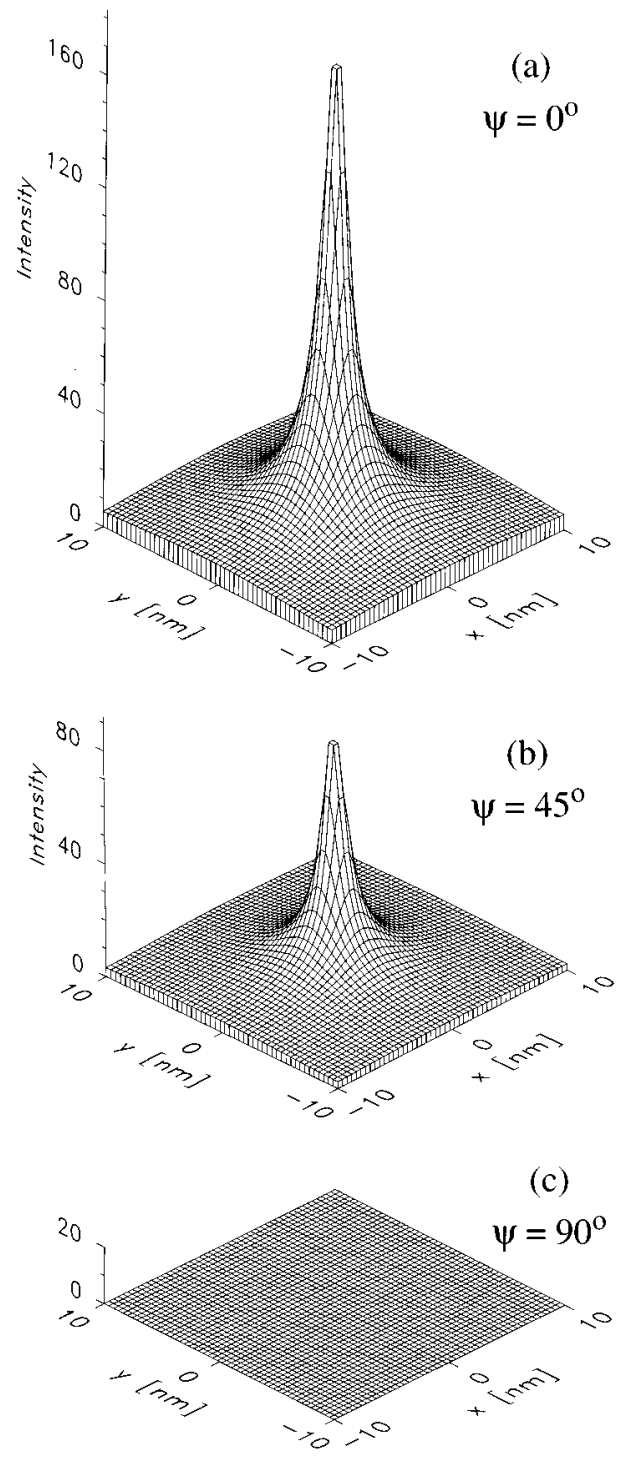

FIG. 2. Total field intensity in the tip-surface junction, at an altitude $z=4[\mathrm{~nm}]$; (a) p polarization, (b) intermediate polarization $\left(\psi=45^{\circ}\right)$, (c) $\mathrm{s}$ polarization. The angle of incidence is $\theta=80^{\circ}$.

times the intensity of the incident field [Fig. 2(a)]. This enhancement factor strongly depends on the polarization of the incident field: for $\psi=45^{\circ}$, its value decreases to 92 [Fig. 2(b)] and for $\psi=90^{\circ}$ (s polarization), the total field intensity drops to zero and the field distribution in the junction looks perfectly flat [Fig. 2(c)]. Actually, $I$ depends on the square of $\cos (\psi)$, which emphasizes the dominating role played by the vertical field component in the scattering at the tip apex. Also note in Fig. 2 the extremely strong lateral confinement of the field below the tip apex. This strong lateral confinement is also observed when $\psi \neq 90^{\circ}$ [Fig. 2(b)].

The total field intensity $I$ depends also on the altitude $z$ in the junction. For $\mathrm{p}$ polarization, $I$ grows overexponentially when approaching the very tip, giving enhancement factors of a few hundred. Let us note here that if we consider a physical system that supports plasmons (e.g., silver tip and surface as in Ref. 9) we obtain intensity enhancements one order of magnitude higher than in the present system.

For s polarization, we observe minute variations of $I$

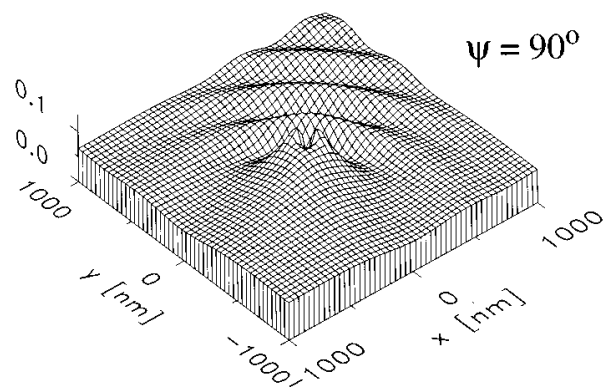

FIG. 3. Same results as in Fig. 2(c) (s polarization), but with a larger computation window and an intensity scale enlarged 20000 times.

with $z$. On the other hand, quite a subtle behavior of the field for $\mathrm{s}$ polarization is revealed in Fig. 3, which shows the results presented in Fig. 2(c) on a much enlarged scale. The two peaks observed in Fig. 3 are caused by the scattering at the two front facets of the tetragonal tip (the illumination angle $\varphi=45^{\circ}$ is such that these two front facets are equally illuminated for s polarization). When the field is computed at larger distances from the tip (e.g., for $z=3[\mathrm{~nm}]$ ), these peaks disappear and there only remains a depletion below the tip apex. Notice also in Fig. 3 the scattered wave behind the tip.

Another important experimental parameter is the angle of incidence $\theta$ (Fig. 1). From the preceding results, where it was shown that the vertical field component plays a dominant role, one would expect a maximum enhancement when $\theta \rightarrow 90^{\circ}$, as this maximizes the vertical field components. In fact, it turns out that it is not at all the case, as is evident in Fig. 4, where we report the total field intensity as a function of the angle of incidence $\theta$. We observe in this figure that for $\mathrm{p}$ polarization, the intensity in the junction is maximum for $\theta=59^{\circ}$ and tends to zero when $\theta \rightarrow 90^{\circ}$. This behavior is due to the proximity of the surface. Indeed, although the amplitude of the reflected field is proportional to $\theta$, its phase is opposed to that of the incident field. Thus, just at the surface, the total field decreases when $\theta$ increases. The position of the maximum observed in Fig. 4 is extremely close to the value $\theta_{\max }$ obtained by maximizing the Fresnel solution for the total vertical field for reflection from a bare surface. ${ }^{13}$ It is

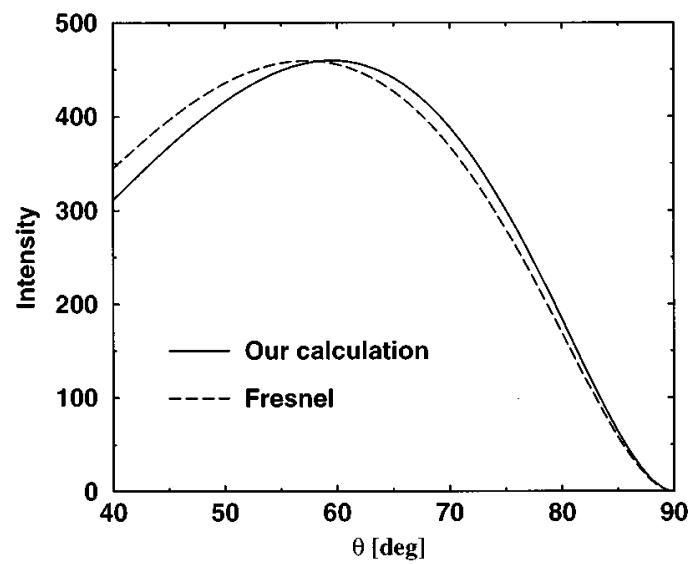

FIG. 4. Total field intensity below the tip apex at an altitude $z=4[\mathrm{~nm}]$, as a function of the angle of incidence $\theta$ (solid line) and intensity of the vertical field calculated with Fresnel theory for the same altitude (dashed line). 
not exactly the same value because $\theta_{\max }$ depends on the height $z$ above the surface where the total field is calculated. ${ }^{13}$ Actually, $\theta_{\max }$ increases with the altitude $z$. As the tip in our calculation has a certain $z$ extension, this explains why the maximum value observed in Fig. 4 is slightly higher than the Fresnel value $\theta_{\max }$ computed for the altitude of the apex.

It is important to point out here two limitations of our model. On the one hand, we used a finite sized tip for the calculation. But calculations with different tip sizes have shown that for the subwavelength tip size that we investigated, the intensity in the junction is proportional to the tip volume. Also, the radius of curvature of the tip apex strongly influences the enhancement and when doubling the radius of curvature of the apex, the enhancement dropped by a factor of about 2. Let us note that the influence of the apex on the field in the junction can be investigated with a twodimensional model, as presented recently in detail by Madrazo et al. ${ }^{14}$

On the other hand, we described the metal in the tip apex by a local dielectric function. Now, at a very small distance from the metal, nonlocal effects can become important. These effects are known to screen the electronic excitations in the metal which would lead to weaker enhancement factors outside the metal. ${ }^{15}$

Finally, we believe that accurate control of the strong optical field gradients at a local probe tip apex, as presented in this letter, finds application not only in imaging techniques, but could also be operative for nanoscale manipula- tions where an object is placed at the desorption threshold by conventional means (e.g., voltage bias in STM) and an external optical field is then used to provide the remaining energy for desorption. ${ }^{16}$

It is a pleasure to acknowledge stimulating discussions with G. Dujardin and D.W. Pohl. This work was supported by the Swiss National Science Foundation.

${ }^{1}$ U. Ch. Fischer and D. W. Pohl, Phys. Rev. Lett. 62, 458 (1989)

${ }^{2}$ Y. Inouye and S. Kawata, Opt. Lett. 19, 159 (1994).

${ }^{3}$ F. Zenhausern, M. P. O'Boyle, and H. K. Wickramasinghe, Appl. Phys. Lett. 65, 1623 (1994).

${ }^{4}$ S. Kawata and Y. Inouye, Ultramicroscopy 57, 313 (1995).

${ }^{5}$ F. Zenhausern, Y. Martin, and H. K. Wickramasinghe, Science 269, 1083 (1995).

${ }^{6}$ P. Gleyzes, A. C. Boccara, and R. Bachelot, Ultramicroscopy 57, 318 (1995).

${ }^{7}$ R. Bachelot, P. Gleyzes, and A. C. Boccara, Opt. Lett. 20, 1924 (1995).

${ }^{8}$ J. Jersch and K. Dickmann, Appl. Phys. Lett. 68, 868 (1996).

${ }^{9}$ W. Denk and D. W. Pohl, J. Vac. Sci. Technol. B 9, 510 (1991).

${ }^{10}$ J. H. Weaver, C. Krafka, D. W. Lynch, and E. E. Koch, 'Optical properties of Metals,' in Physics Data (Fachinformationszentrum, Karlsruhe, 1981), Vol. 18-1.

${ }^{11}$ Handbook of Optics, Vol. 2, edited by M. Bass (McGraw-Hill, Optical Society of America, New York, 1995), Chap. 33

${ }^{12}$ O. J. F. Martin, C. Girard, and A. Dereux, Phys. Rev. Lett. 74, 526 (1995).

${ }^{13}$ M. Born and E. Wolf, Principles of Optics, 6th ed. (Pergamon, Oxford, 1980).

${ }^{14}$ A. Madrazo, M. Nieto-Vesperinas, and N. García, Phys. Rev. B 53, 3654 (1996).

${ }^{15}$ C. Girard and F. Hache, Mol. Phys. 70, 811 (1990).

${ }^{16}$ C. Girard, A. Dereux, and O. J. F. Martin, Phys. Rev. B 49, 13872 (1994). 